\title{
Fraction of Electrons Consumed in Electron Acceptor Reduction and Hydrogen Thresholds as Indicators of Halorespiratory Physiology
}

\author{
FRANK E. LÖFFLER, ${ }^{1} \dagger$ JAMES M. TIEDJE, ${ }^{1}$ AND ROBERT A. SANFORD ${ }^{2 *}$ \\ Center for Microbial Ecology, Michigan State University, East Lansing, Michigan 48824-1325, ${ }^{1}$ and Department of \\ Civil and Environmental Engineering, University of Illinois, Urbana, Illinois 61801-2352 2
}

Received 21 December 1998/Accepted 22 June 1999

\begin{abstract}
Measurements of the hydrogen consumption threshold and the tracking of electrons transferred to the chlorinated electron acceptor $\left(f_{e}\right)$ reliably detected chlororespiratory physiology in both mixed cultures and pure cultures capable of using tetrachloroethene, cis-1,2-dichloroethene, vinyl chloride, 2-chlorophenol, 3-chlorobenzoate, 3-chloro-4-hydroxybenzoate, or 1,2-dichloropropane as an electron acceptor. Hydrogen was consumed to significantly lower threshold concentrations of less than $0.4 \mathrm{ppmv}$ compared with the values obtained for the same cultures without a chlorinated compound as an electron acceptor. The $f_{e}$ values ranged from 0.63 to 0.7 , values which are in good agreement with theoretical calculations based on the thermodynamics of reductive dechlorination as the terminal electron-accepting process. In contrast, a mixed methanogenic culture that cometabolized 3-chlorophenol exhibited a significantly lower $f_{e}$ value, 0.012 .
\end{abstract}

Halogenated aliphatic and aromatic compounds are major environmental pollutants (17). Since many contaminated sites are anaerobic, stimulation of reductive dehalogenation is a very promising strategy for bioremediating aquifers and sediments contaminated with halogenated compounds. Reductive dechlorination is a cometabolic or respiratory process that is carried out by organochlorine-reducing bacteria (OCRBs). One example of cometabolism is the reductive dechlorination of tetrachloroethene (PCE) by sulfate reducers $(6,46)$, methanogenic archaea $(16,23)$, or acetogens $(44)$. The dechlorination rates of the cometabolic processes, however, are low, and their contributions to the dehalogenation reactions observed in natural environments are probably negligible. In contrast, respiratory OCRBs that use chlorinated aliphatic and/or aromatic organic compounds as electron acceptors in energy metabolism and hence for growth are likely to be major contributors to microbially mediated dehalogenation reactions in anaerobic environments. The common characteristic of respiratory organohalogen-reducing bacteria is their ability to couple the energy released during reductive dehalogenation to growth in a process known as halorespiration (or chlororespiration for chlorinated chemicals).

The energetics of the terminal electron-accepting process (TEAP) have been shown to control the minimum $\mathrm{H}_{2}$ concentration that can be consumed (i.e., the threshold) $(2,8,9,32$, 33). The $\mathrm{H}_{2}$ threshold is inversely correlated with changes in Gibbs free energy $\left(\Delta G^{0^{\prime}}\right)$ and the electrochemical potential of the $\mathrm{H}_{2}$-consuming reaction (Table 1$)(8,32,33,52)$. Lovley and Goodwin (33) have demonstrated that the steady-state $\mathrm{H}_{2}$ threshold concentrations are controlled by the physiological characteristics of the $\mathrm{H}_{2}$-consuming organisms and are independent of the kinetics of the $\mathrm{H}_{2}$-producing or $\mathrm{H}_{2}$-consuming reactions. This model predicts that the steady-state threshold concentrations of $\mathrm{H}_{2}$ follow the following order (from least to

\footnotetext{
* Corresponding author. Mailing address: Department of Civil and Environmental Engineering, University of Illinois, 3230C Newmark Civil Engineering Laboratory, 205 North Mathews, Urbana, IL 618012352. Phone: (217) 244-7250. Fax: (217) 333-6968. E-mail: rsanford @uiuc.edu.

$\dagger$ Present address: School of Civil and Environmental Engineering, Georgia Institute of Technology, Atlanta, GA 30332-0512.
}

most energetically favorable TEAP): acetogenesis $>$ methanogenesis $>$ sulfate reduction (sulfidogenesis) $>$ Fe(III) reduction $>\mathrm{Mn}(\mathrm{IV})$ reduction $>$ denitrification. Based on the threshold model, one would predict that chlororespiring OCRBs should consume $\mathrm{H}_{2}$ so that the threshold concentrations are lower than the threshold concentrations of acetogens, methanogens, and sulfate reducers and probably very close to the threshold concentrations of organisms that exhibit dissimilatory reduction of nitrate to ammonia (ammonifiers).

Another method used to identify the activity of chlororespiring organisms is to determine $f_{e}$ or $f_{s} . f_{e}$ is the fraction of electrons used for energy formation (i.e., the fraction of electrons released during oxidation of the electron donor that are directed toward reduction of the terminal electron acceptor). The fraction of electrons derived from the electron donor that is incorporated into biomass is the synthesis fraction $\left(f_{s}\right)(10$, 36). Since $f_{e}+f_{s}=1$, and $f_{s(\max )}$ is directly related to the maximum growth yield, $f_{e}$ can be calculated from the maximum growth yield, when it is available. While accurate determinations of growth yields require the use of pure cultures, $f_{e}$ values can be experimentally determined for pure and mixed cultures. For example, estimated $\mathrm{f}_{\mathrm{e}}$ values for chlororespiring OCRBs have been predicted to be very similar to $f_{e}$ values for ammonifiers according to thermodynamics-based models $(20,21,36)$.

Despite the potential importance of chlororespiring OCRBs in reductive dechlorination processes in anaerobic environments, no convenient methods to assay this activity have been developed. So far, the only method which conclusively demonstrates that chlororespiration occurs involves isolating the dechlorinating populations in pure culture and showing that growth with an electron donor that does not support substrate level phosphorylation is dependent on the dechlorination reaction. This approach is time-consuming, since isolating halorespiring OCRBs in pure culture can be tedious. Many of these organisms grow slowly or require additional growth factors, such as amino acids and vitamins $(11,22,35)$. For example, despite extensive efforts to isolate vinyl chloride (VC)- and polychlorinated biphenyl-respiring OCRBs, to date no pure cultures that grow in this manner have been obtained.

Here we present evidence that both measuring the $\mathrm{H}_{2}$ threshold and determining $\mathrm{f}_{\mathrm{e}}$ provide excellent indications of halorespiratory physiology. The applicability of both methods 
TABLE $1 . \mathrm{H}_{2}$ threshold concentrations, $\Delta \mathrm{G}^{0^{\prime}}$ values, and redox potentials of different redox couples with $\mathrm{H}_{2}$ as the electron donor

\begin{tabular}{|c|c|c|c|c|c|}
\hline \multirow{2}{*}{ TEAP } & \multicolumn{2}{|c|}{$\mathrm{H}_{2}$ threshold concn } & \multirow{2}{*}{$\begin{array}{c}\Delta G^{0^{\prime}}(\mathrm{kJ} / \mathrm{mol} \\
\left.\quad \text { of } \mathrm{H}_{2}\right)^{a}\end{array}$} & \multirow{2}{*}{$\mathrm{E}_{0}{ }^{\prime}(\mathrm{V})^{b}$} & \multirow{2}{*}{ Reference(s) } \\
\hline & ppmv & $\mathrm{nM}$ & & & \\
\hline Acetogenesis & $430-4,660$ & $336-3,640$ & -26.1 & -0.28 & 2,9 \\
\hline Methanogenesis & $6-120$ & $5-95$ & -33.9 & -0.24 & $8,9,32,33$ \\
\hline Sulfate reduction $\left(\mathrm{SO}_{4}{ }^{2-} \rightarrow \mathrm{HS}^{-}\right)$ & $1.3-19$ & $1-15$ & -38.0 & -0.22 & $8,9,33$ \\
\hline Fumarate reduction & 0.02 & 0.015 & -86.2 & +0.032 & 9 \\
\hline Ammonification $\left(\mathrm{NO}_{3}{ }^{-} \rightarrow \mathrm{NH}_{4}{ }^{+}\right)$ & $0.02-0.03$ & $0.015-0.025$ & -149.9 & +0.36 & 8,9 \\
\hline Nitrate reduction $\left(\mathrm{NO}_{3}{ }^{-} \rightarrow \mathrm{N}_{2} \mathrm{O}, \mathrm{N}_{2}\right)$ & $<0.06$ & $<0.05$ & -240 & +0.79 & 33,34 \\
\hline $\mathrm{Fe}(\mathrm{III})$ reduction & $0.13-1$ & $0.1-0.8$ & -228.3 & +0.77 & $8,33,34$ \\
\hline Chlororespiration & $<0.4^{c}$ & $<0.3^{c}$ & -130 to -187 & 0.30 to 0.55 & This study \\
\hline
\end{tabular}

${ }^{a}$ The $\Delta G^{0^{\prime}}$ values were obtained from references 13 through 15 and 45 or were calculated from free energies of formation.

${ }^{b}$ The redox potentials $\left(\mathrm{E}_{0}{ }^{\prime}\right)$ were calculated by using the relationship $\mathrm{E}_{0}{ }^{\prime}=\left(\Delta G^{0^{\prime}} /-0.193\right)-414(14,15,47)$.

${ }^{c}$ Data from this study.

was demonstrated with several pure and mixed cultures. Furthermore, $\mathrm{H}_{2}$ threshold measurements obtained for mixed cultures that dechlorinated VC to ethene (ETH) indicated that VC-respiring OCRBs were present, despite the fact that we were not able to obtain these organisms in pure culture.

\section{MATERIALS AND METHODS}

Chemicals. The following chlorinated compounds were used in this study: PCE, trichloroethene, cis-1,2-dichloroethene (cis-DCE), 1,2-dichloropropane (1,2-D), 3-chlorobenzoate (3-CBA), 3-chloro-4-hydroxybenzoate (3Cl-4-HBA), 2-chlorophenol (2-CP), 3-chlorophenol (3-CP), and 2,5-dichlorophenol (2,5DCP), all of which were obtained from Aldrich, Milwaukee, Wis.; and VC, which was obtained from Fluka Chemical Corp., Ronkonkoma, N.Y.

Organisms, medium preparation, and growth conditions. The following pure cultures were used in this study: Desulfitobacterium chlororespirans DSM 11544 or ATCC 700175 (27, 39), Desulfitobacterium sp. strain Viet1 (29), Desulfuromonas sp. strain BB1 (30), Desulfomonile tiedjei ATCC 49306 (11, 43), and strain 2CP-C (38), a close relative of strain 2CP-1 (5). The 2,5-DCP-dechlorinating OM enrichment culture was derived from chlorophenol-contaminated soil (38), and the 3-CP-dechlorinating methanogenic enrichment culture was obtained from residential compost in the Lansing area of Michigan (40). Chloroethene-dechlorinating mixed cultures were derived from freshwater river sediments obtained from the King Salmon River in Alaska (culture KS), the Red Cedar River in Michigan (cultures RC), the Au Sable River in Michigan (cultures AuS), and the Père Marquette River in Michigan (cultures PM) $(28,29)$. Cultures KS 1,2-D $_{\text {, }}$ and $\mathrm{RC}_{1,2-\mathrm{D}}$ were enriched for the ability to dechlorinate 1,2-D to propene, and cultures RC, AuS, and PM were enriched for the ability to dechlorinate PCE to ETH. Subcultures of the cultures that dechlorinated PCE to ETH were established after sediment-free, nonmethanogenic cultures had been obtained (29). These subcultures were fed with PCE $(20 \mu \mathrm{mol})$, cis-DCE $(26.5 \mu \mathrm{mol})$, or VC $(41 \mu \mathrm{mol})$. Cultures were transferred when approximately $20 \mu \mathrm{mol}$ of the initial chlorinated compound had been dechlorinated. All subcultures had undergone at least 10 serial transfers $\left(1 \%\right.$ [vol/vol] inoculum) at the time that the $\mathrm{H}_{2}$ threshold measurements were initiated.

Reduced anaerobic mineral medium was prepared as described previously (27, $28,39)$. To obtain $\mathrm{f}_{\mathrm{e}}$ measurements for cultures of Desulfitobacterium chlororespirans, the $\mathrm{OM}$ enrichment culture, and the 3-CP-dechlorinating mixed methanogenic culture, the medium described by Sanford et al. (39) was used. The same medium was used to monitor $\mathrm{H}_{2}$ consumption in cultures of strain $2 \mathrm{CP}-\mathrm{C}$. For most other experiments the medium described by Löfler et al. $(27,28)$ was used; the only exceptions were the experiments in which $\mathrm{f}_{\mathrm{e}}$ was measured in strain BB1 and Viet1 cultures. In these experiments, dechlorination was quantified by measuring the increase in the chloride ion concentration, and a chloride-free medium was used. This medium contained (per liter) $0.5 \mathrm{~g}$ of $\mathrm{MgSO}_{4} \cdot 6 \mathrm{H}_{2} \mathrm{O}, 0.3 \mathrm{~g}$ of $\mathrm{NH}_{4} \mathrm{SO}_{4}, 0.3 \mathrm{~g}$ of $\mathrm{KSO}_{4}, 0.015 \mathrm{~g}$ of $\mathrm{CaSO}_{4} \cdot \mathrm{H}_{2} \mathrm{O}, 0.05 \mathrm{mg}$ of resazurin, $1 \mathrm{ml}$ of trace element solution A (27), $1 \mathrm{ml}$ of trace element solution B (27), $0.048 \mathrm{~g}$ of $\mathrm{Na}_{2} \mathrm{~S} \cdot 9 \mathrm{H}_{2} \mathrm{O}, 0.035 \mathrm{~g}$ of L-cysteine, and $2.52 \mathrm{~g}$ of $\mathrm{NaHCO}_{3}$. After autoclaving, 2 $\mathrm{mM}$ potassium phosphate buffer $(\mathrm{pH} 7.2)$ and a vitamin solution (27) were added. Cultures were incubated in $160-\mathrm{ml}$ (nominal capacity) serum bottles containing $100 \mathrm{ml}$ of medium (referred to below as $100-\mathrm{ml}$ cultures). All serum bottles were incubated in the dark at $25^{\circ} \mathrm{C}$ with the butyl rubber stopper down without shaking. Undiluted halogenated compounds were added to cultures with 5- $\mu$ l Hamilton syringes equipped with Chaney adapters (Hamilton, Reno, Nev.) in order to achieve reproducibility. Gaseous VC was added by syringe. Chlorinated benzoates and phenols were added from anaerobic, filter-sterilized stock solutions to final concentrations of 2 and $0.2 \mathrm{mM}$, respectively. Dechlorination was monitored as described below, and cultures were respiked with the appropriate chlorinated compounds before they were completely depleted. $\mathrm{H}_{2}$ was added by syringe at the mixing ratios indicated below. Molar gas concentrations were calculated by using a molar volume of 24.48 liters at $25^{\circ} \mathrm{C}$.

Experimental determination of $\mathbf{f}_{e}$ and $\mathbf{f}_{s}$ values. $f_{e}$ values were determined in cultures containing a chlorinated compound and bicarbonate as the only electron acceptors and either $\mathrm{H}_{2}$ plus acetate $(85,000 \mathrm{ppm}$ by volume [ppmv] and $1 \mathrm{mM}$, respectively; OM enrichment culture), acetate (5 mM; strains BB1 and 2CP-C), lactate ( $5 \mathrm{mM}$; Desulfitobacterium sp. strain Viet1 and Desulfitobacterium chlororespirans), or a volatile fatty acid (VFA) mixture $(625 \mu \mathrm{M}$ formate, $125 \mu \mathrm{M}$ succinate, $125 \mu \mathrm{M}$ propionate, and $125 \mu \mathrm{M}$ butyrate; methanogenic enrichment culture) as the only electron donor(s). For studies of BB1 and Viet1 cultures, PCE was added by syringe from anaerobic, autoclaved, hexadecane stock solutions ( $125 \mu \mathrm{l}$ PCE in $10 \mathrm{ml}$ of hexadecane per $100-\mathrm{ml}$ culture). The $\mathrm{f}_{\mathrm{e}}$ values were determined by linear regression analysis by plotting the amount of electron pairs released during electron donor oxidation as $2[\mathrm{H}]$ against the amount of electron pairs used to reduce the chlorinated electron acceptor. $\mathrm{f}_{\mathrm{e}}$ was derived from the slope of the regression line. In most cases at least three cultures were used to determine $f_{e}$; the only exceptions were the methanogenic enrichment cultures, which were analyzed in duplicate. The cultures were each started with a $1 \%$ ( vol/vol) inoculum, and $0.5-\mathrm{ml}$ samples were periodically withdrawn (every 2 to 3 days) and analyzed to determine chloride release and electron donor consumption. Aromatic dechlorination products and VFA were quantified by high-performance liquid chromatography (HPLC), and PCE dechlorination was quantified by determining the amount of chloride ions released into the medium by using the calorimetric assay described below. Electron donor (lactate) consumption in cultures of Desulfitobacterium chlororespirans and Desulfitobacterium sp. strain Viet1 was examined by measuring the amount of acetate formed during incomplete oxidation of lactate.

Hydrogen threshold measurements. $\mathrm{H}_{2}$ partial pressures were measured in the headspaces of cultures incubated at $25^{\circ} \mathrm{C}$ and are expressed below in parts per million by volume $\left(1 \mathrm{ppmv}=\mathrm{ca} .0 .1 \mathrm{~Pa}=\mathrm{ca} \cdot 10^{-6} \mathrm{~atm}\right)$ according to the convention adapted by Conrad (8). In order to compare our data to the data obtained in other studies, dissolved $\mathrm{H}_{2}$ concentrations were calculated by using the following equation: $\mathrm{H}_{2}$ (dissolved) $=L P / R T$, where $\mathrm{H}_{2}$ (dissolved) is the aqueous concentration of $\mathrm{H}_{2}$ (in moles per liter); $L$ is the Ostwald coefficient for $\mathrm{H}_{2}$ solubility, which is 0.01913 at $25^{\circ} \mathrm{C}(49) ; R$ is the universal gas constant $\left(0.0821\right.$ liter $\cdot$ atm K $\left.{ }^{-1} \mathrm{~mol}^{-1}\right) ; P$ is the pressure (in atmospheres); and $T$ is the kelvin temperature $(3,34)$. Duplicate $100 \mathrm{ml}$-cultures were inoculated $(1 \%$, $\mathrm{vol} / \mathrm{vol}$ ) from dechlorinating cultures that had completely reduced all of the chlorinated electron acceptor present. One set of the duplicate cultures was amended with the individual chlorinated compounds, and the other set did not receive an electron acceptor other than bicarbonate. The concentrations of chlorinated compounds were determined weekly, and the $\mathrm{H}_{2}$ concentration was measured every third week. Cultures that received a chlorinated compound were spiked with the same chlorinated electron acceptor before it was completely depleted. Values for $\mathrm{H}_{2}$ thresholds were assessed when the $\mathrm{H}_{2}$ concentration remained stable (i.e., when there was a less than $5 \%$ decrease over a 6 -week period). After the $\mathrm{H}_{2}$ concentrations reached constant threshold values, $\mathrm{H}_{2}$ (500 to $10,000 \mathrm{ppmv}$ ) was added to the cultures, and consumption of this $\mathrm{H}_{2}$ to a constant threshold value was monitored again. $\mathrm{H}_{2}$ thresholds in pure cultures (except strain 2CP-C cultures) were determined by using the same protocol, except that acetate was replaced by $20 \mathrm{mM}$ pyruvate. The cultures were grown on pyruvate with and without the appropriate chlorinated electron acceptors. 3-CBA (2 mM), 3Cl-4-HBA (2 mM), and PCE $(0.2 \mathrm{mM})$ were used as electron acceptors for Desulfomonile tiedjei, Desulfitobacterium chlororespirans, and Desulfitobacterium sp. strain Viet1, respectively. All cultures were initiated with $1 \%$ ( $\mathrm{vol} / \mathrm{vol}$ ) inocula from cultures that had undergone at least three transfers under fermenting or chlororespiring growth conditions. When the initial amount of chlorinated electron acceptor was depleted, the cultures were fed with $2 \mathrm{mM}$ 3-CBA, with $2 \mathrm{mM} 3 \mathrm{Cl}-4-\mathrm{HBA}$, or with PCE (50 $\mu \mathrm{l}$ in $5 \mathrm{ml}$ of hexadecane). Strain 2CP-C utilized acetate or $\mathrm{H}_{2}$ as an electron donor for reductive dechlo- 

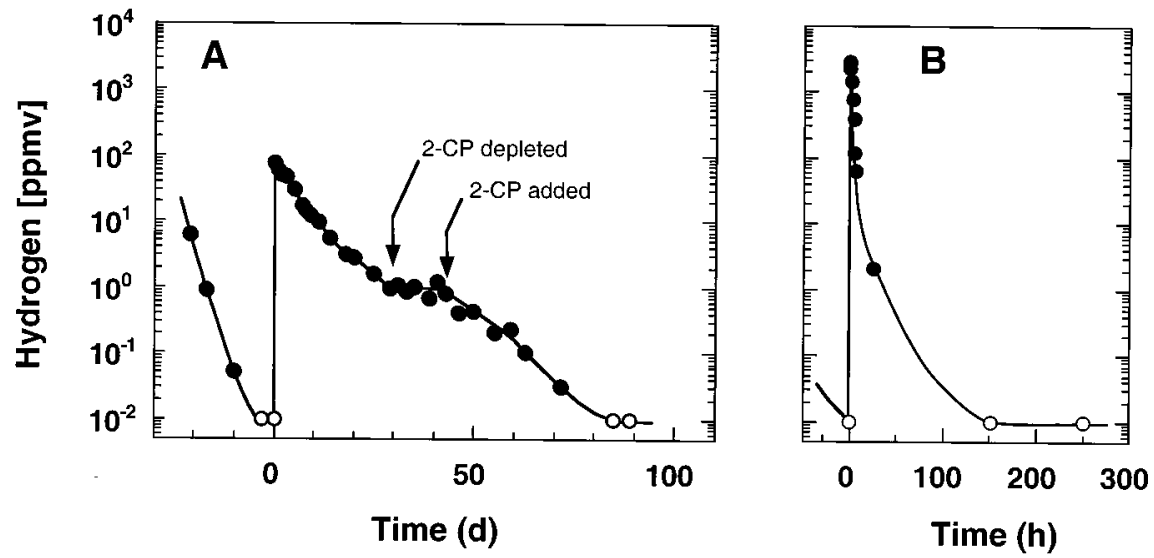

FIG. $1 . \mathrm{H}_{2}$ consumption by strain $2 \mathrm{CP}-\mathrm{C}$ with $2-\mathrm{CP}$ as the electron acceptor and $\mathrm{H}_{2}$ as the electron donor. (A) Two freshly inoculated cultures containing $0.2 \mathrm{mM}$ acetate were fed 2-CP. After the $\mathrm{H}_{2}$ concentration was less than $0.01 \mathrm{ppmv}$, the cultures were fed $100 \mathrm{ppmv}$ of $\mathrm{H}_{2}$. 2-CP was respiked when it was depleted, except for the 14-day period indicated by the arrows. (B) Triplicate cultures were initially fed $2 \mathrm{mM}$ acetate and 2-CP. After $4 \mathrm{mM} 2-\mathrm{CP}$ had been added, all of the acetate was consumed and the $\mathrm{H}_{2}$ threshold concentrations were less than 0.01 ppmv. The cultures were then fed 2-CP and 2,500 ppmv of $\mathrm{H}_{2}$, and $\mathrm{H}_{2}$ consumption was monitored again. Note the logarithmic scale of the $y$ axis. The detection limit for $\mathrm{H}_{2}$ was 0.01 ppmv, and the open circles indicate data that could not be quantified due to the limitations of the detection method. The data were averaged, and the variability between cultures was less than $20 \%$. No $\mathrm{H}_{2}$ consumption was observed in cultures that did not receive $2-\mathrm{CP}$.

rination of 2-CP. This strain was grown with acetate $(0.2$ or $2 \mathrm{mM})$ and $0.2 \mathrm{mM}$ $2-\mathrm{CP}$, and the culture was respiked with $2-\mathrm{CP}(0.2 \mathrm{mM})$ when the $2-\mathrm{CP}$ was depleted. When constant $\mathrm{H}_{2}$ threshold concentrations were reached, the cultures were spiked with 100 or 2,500 ppmv of $\mathrm{H}_{2}$, and the consumption of $\mathrm{H}_{2}$ was monitored again.

Analytical methods. All volatile chlorinated aliphatic compound contents were measured in the headspaces of $100-\mathrm{ml}$ cultures at $25^{\circ} \mathrm{C}$ and were analyzed by using a gas chromatograph and direct (splitless) injection (28). Gas-tight 0.25-ml syringes (Hamilton) with Teflon push-button valves were used to withdraw and inject $0.2-\mathrm{ml}$ gas samples (19).

$\mathrm{H}_{2}$ contents were measured with a Hewlett-Packard gas chromatograph (model series 6890) equipped with a reduction gas detector (Trace Analytical, Menlo Park, Calif.). Headspace samples $(3 \mathrm{ml})$ were injected into a $1-\mathrm{ml}$ gas sampling loop and were separated with a molecular sieve analytical column (MS 5A; $0.79 \mathrm{~m}$ by $0.3175 \mathrm{~cm}$; $60 / 80 \mathrm{mesh}$; Trace Analytical) at an oven temperature of $40^{\circ} \mathrm{C}$. Ultra-high-purity nitrogen ( $99.999 \%$ pure; AGA Gas, Inc., Maumee, Ohio) was used as the carrier gas at a flow rate of $27.5 \mathrm{ml} \mathrm{min}{ }^{-1}$ after it was passed through a catalytic combustion converter (Trace Analytical) to remove traces of $\mathrm{H}_{2}$. The detection limit for $\mathrm{H}_{2}$ under these conditions was 0.01 ppmv (ca. 0.001 $\mathrm{Pa}$ ). Samples containing 50 to $500 \mathrm{ppmv}$ (ca. 5 to $50 \mathrm{~Pa}$ ) of $\mathrm{H}_{2}$ were diluted in $\mathrm{H}_{2}$-free dinitrogen immediately before they were injected, or the volume of the gas sampling loop was reduced to $0.25 \mathrm{ml} \mathrm{H}_{2}$ concentrations greater than 500 ppmv (ca. $50 \mathrm{~Pa}$ ) were determined by using a thermal conductivity detector as described previously (28). Analyses of aromatic compounds and VFAs were performed by HPLC $(27,39)$. Chloride release was measured by a colorimetric assay (1).

\section{RESULTS}

$\mathbf{H}_{2}$ threshold. To test the $\mathrm{H}_{2}$ threshold concept, we evaluated the minimum $\mathrm{H}_{2}$ contents obtained in the presence and in the absence of various chlorinated electron acceptors with a variety of pure and mixed cultures. $\mathrm{H}_{2}$ was repeatedly consumed until the concentration was less than 0.01 ppmv by strain 2CP-C as long as 2-CP was present regardless of whether acetate or $\mathrm{H}_{2}$ was added at high or low concentrations (Fig. 1). In contrast, cultures that had dechlorinated all of the 2-CP to phenol (all of the chlorinated electron acceptor was reduced) did not consume $\mathrm{H}_{2}$ at all. Therefore, the fate of the chlorinated electron acceptor was closely monitored in all other experiments to ensure that the consumption of $\mathrm{H}_{2}$ was never limited by electron acceptor availability. Desulfitobacterium chlororespirans and Desulfitobacterium sp. strain Viet1, which are known to be chlororespiring microorganisms (27, 29, 39; unpublished data), reduced the $\mathrm{H}_{2}$ threshold concentrations to $0.27 \pm 0.06$ ppmv $(n=4)$ and $0.4 \pm 0.13$ ppmv $(n=6)$, respectively, when they were grown with $3 \mathrm{Cl}-4-\mathrm{HBA}$ or PCE as an electron acceptor. Desulfomonile tiedjei DCB-1, another chlororespirer (37), reduced the $\mathrm{H}_{2}$ concentration below the detection limit ( $0.01 \mathrm{ppmv})$ when it was grown with 3-CBA as the electron acceptor; this concentration was considerably less than the 0.7 ppmv previously reported for DCB-1 (12). In contrast, the same cultures exhibited significantly higher $\mathrm{H}_{2}$ threshold concentrations (range, 100 to $400 \mathrm{ppmv}$ ) when they were grown with pyruvate fermentation in the absence of a chlorinated compound.

We also monitored the $\mathrm{H}_{2}$ concentrations in several acetogenic and nonmethanogenic mixed cultures that dechlorinated different chloroethenes or 1,2-D. The absence of methanogenic archaea was confirmed by the lack of methane production from methanogenic substrates, and no archaeal 16S ribosomal DNA could be amplified by PCR from genomic DNA isolated from these cultures (29). Reductive dechlorination in all of the mixed cultures tested was supported by $\mathrm{H}_{2}$, and in all of the mixed cultures $\mathrm{H}_{2}$ was consumed to threshold concentrations below 0.4 ppmv in the presence of individual chlorinated aliphatic compounds (Table 2). Interestingly, the three VC-dechlorinating cultures also consumed $\mathrm{H}_{2}$ to concentrations of 0.19 to $0.21 \mathrm{ppmv}$, indicating that $\mathrm{VC}$ may be used as a terminal electron acceptor. In the absence of a chlorinated compound as the electron acceptor, the same cultures consumed $\mathrm{H}_{2}$ to threshold concentrations ranging from 250 to 440 ppmv, indicating that acetogenesis was the TEAP. Formation of acetate from $\mathrm{H}_{2}-\mathrm{CO}_{2}$ in all of the mixed cultures was confirmed by HPLC analyses. Only the $\mathrm{KS}_{1,2-\mathrm{D}}$ enrichment culture reduced the $\mathrm{H}_{2}$ concentration to about 120 ppmv when it was grown acetogenically without $1,2-\mathrm{D}$, but this value was still 2 orders of magnitude higher than the threshold value observed for the same culture grown in the presence of the chlorinated electron acceptor.

Determination of $\mathbf{f}_{\mathbf{e}}$ values. $f_{e}$ values were determined experimentally by using several pure and mixed cultures capable of carrying out at least one reductive dechlorination reaction (Fig. 2). The total amounts of electron donor oxidized and electron acceptor reduced in replicate cultures varied less than $25 \%$, and the differences in $\mathrm{f}_{\mathrm{e}}$ values determined for identical cultures were less than $5 \%$. The $\mathrm{f}_{\mathrm{e}}$ values for the chlororespiring organisms Desulfitobacterium chlororespirans, Desulfitobac- 
TABLE $2 . \mathrm{H}_{2}$ thresholds exhibited by cultures in the presence or in the absence of chlorinated aliphatic compounds supplied as electron acceptors

\begin{tabular}{llcc}
\hline & & \multicolumn{2}{c}{$\mathrm{H}_{2}$ concn $(\mathrm{ppmv})^{b}$} \\
\cline { 3 - 4 } Culture & Compound $^{a}$ & $\begin{array}{c}\text { In the absence of } \\
\text { chlorinated elec- } \\
\text { tron acceptor }^{c}\end{array}$ & $\begin{array}{c}\text { In the presence of } \\
\text { chlorinated elec- }^{\text {tron acceptor }}{ }^{d}\end{array}$ \\
\hline KS & 1,2-D & $117.3 \pm 37.1(7)^{e}$ & $0.30 \pm 0.14(15)$ \\
RC & 1,2-D & $343.2 \pm 62.7(4)$ & $0.25 \pm 0.17(23)$ \\
PM & PCE & $319.3 \pm 112.4(3)$ & $0.21 \pm 0.16(5)$ \\
PM & cis-DCE & $438.7 \pm 84.7(3)$ & $0.24 \pm 0.07(6)$ \\
PM & VC & $379.3 \pm 117.3(3)$ & $0.20 \pm 0.05(7)$ \\
AuS & PCE & $318.8 \pm 85.7(5)$ & $0.21 \pm 0.16(11)$ \\
AuS & cis-DCE & $368.3 \pm 88.3(4)$ & $0.34 \pm 0.25(6)$ \\
AuS & VC & $253.2 \pm 60.9(4)$ & $0.19 \pm 0.13(8)$ \\
RC & PCE & $334.1 \pm 24.5(7)$ & $0.16 \pm 0.07(10)$ \\
RC & cis-DCE & $413.3 \pm 107(3)$ & $0.21 \pm 0.08(9)$ \\
RC & VC & $369 \pm 61.7(3)$ & $0.21 \pm 0.11(13)$ \\
\hline
\end{tabular}

${ }^{a}$ Compound with which the culture was enriched.

${ }^{b} \mathrm{H}_{2}$ concentrations were monitored until stable threshold values were obtained. When no further decrease in the $\mathrm{H}_{2}$ concentration was observed, a cultures was fed $\mathrm{H}_{2}$ (500 to 10,000 ppmv), and the consumption of $\mathrm{H}_{2}$ to a constant threshold value was monitored again. Two or three threshold concentrations were obtained per culture.

${ }^{c}$ Bicarbonate was supplied as the only available electron acceptor, and initially $5 \mathrm{ml}(83,000 \mathrm{ppmv})$ of $\mathrm{H}_{2}$ was added as the electron donor.

${ }^{d}$ Cultures were grown in a bicarbonate-buffered medium in the presence of individual chlorinated aliphatic compounds and $5 \mathrm{ml}(83,000 \mathrm{ppmv})$ of $\mathrm{H}_{2}$ as the electron donor. Headspace analyses to monitor dechlorination were performed weekly, and the chlorinated compounds were respiked before they were completely depleted.

${ }^{e}$ Mean \pm standard deviation. The numbers in parentheses are the numbers of replicate analyses.

terium sp. strain Viet1, Desulfuromonas sp. strain BB1, and strain 2CP-C were $0.67,0.63,0.66$, and 0.64 , respectively. An $\mathrm{f}_{\mathrm{e}}$ value of 0.65 was obtained for the 2,5-DCP-dechlorinating OM enrichment culture. No consumption of acetate was observed in the OM culture, indicating that acetate was not used as an electron donor for 2,5-DCP dechlorination. No loss of $\mathrm{H}_{2}$ was observed in autoclaved controls, indicating that the consumption that occurred prior to dechlorination was biologically mediated (Fig. 2). $f_{e}$ values of 0.6 to 0.7 were also obtained for enrichment cultures derived from a contaminated aquifer in which PCE was the terminal electron acceptor. These enrichment cultures yielded a pure culture capable of using PCE as the electron acceptor. The mixed methanogenic enrichment culture that cometabolized $3-\mathrm{CP}$, in contrast, exhibited $\mathrm{f}_{\mathrm{e}}$ values of less than 0.015 . This culture was transferred several times with no increase in the $f_{e}$ value.

\section{DISCUSSION}

Obtaining proof of the chlororespiratory physiology of OCRBs has often involved a long and laborious set of experiments. After we examined several pure and mixed dechlorinating cultures, it became evident that $\mathrm{H}_{2}$ threshold concentrations and $\mathrm{f}_{\mathrm{e}}$ values are excellent indicators of the presence and activity of chlororespiratory OCRBs.

The $\mathrm{H}_{2}$ threshold concentrations depend on the thermodynamics of the $\mathrm{H}_{2}$-consuming step or, more explicitly, the thermodynamics of the terminal electron-accepting reaction $(9,33$, 52). Due to this relationship the characteristic steady-state $\mathrm{H}_{2}$ threshold concentration indicates which electron acceptor is used in the TEAP (Table 1). According to the threshold model, one would predict that chlororespiring OCRBs consume $\mathrm{H}_{2}$ to levels below those the threshold concentrations observed for acetogens, methanogens, and sulfidogens. Our results confirm the validity of this hypothesis that reductive dechlorination is the TEAP. For example, due to the thermodynamic restrictions of $\mathrm{CO}_{2}$ reduction to acetate, acetogenesis cannot explain the consumption of $\mathrm{H}_{2}$ to the very low concentrations measured in the presence of the chlorinated compounds. Indeed, this is consistent with the observations of Yang and McCarty (50), who found that acetogenesis (and methanogenesis) stopped and dechlorination continued in the presence of cis-DCE once the $\mathrm{H}_{2}$ concentration was less than $11 \mathrm{nM}$ (14 ppmv). The results of Conrad (8) show that TEAPs with highly negative Gibbs free energies (i.e., TEAPs with ferric iron or nitrate used as the electron acceptor) exhibit $\mathrm{H}_{2}$ threshold values similar to those of chlororespiring cultures even though there are different amounts of free energy $\left(\Delta G^{\prime}\right)$ available from the individual terminal electron-accepting reactions. The reasons for this phenomenon are unclear. In the absence of interfering TEAPs, like ferric iron reduction or nitrate reduction, however, it is reasonable to expect that $\mathrm{H}_{2}$ threshold measurements can reliably indicate that chlororespiratory reductive dechlorination is the dominant TEAP. The possible exceptions are microbial populations, such as Desulfuromonas sp. strain TT4B and BB1 populations, which do not use $\mathrm{H}_{2}$ as an electron donor for reductive dechlorination (24, $30,31)$. The relative importance of these types of microorganisms in the environment with regard to the $\mathrm{H}_{2}$ threshold, however, has yet to be established.

Factors other than the thermodynamics of the terminal electron-accepting reaction can influence the apparent $\mathrm{H}_{2}$ threshold concentrations. In this study we used basal salts mineral medium, and the sources of $\mathrm{H}_{2}$ were well-defined. Besides gaseous $\mathrm{H}_{2}$, acetate was the only possible source of $\mathrm{H}_{2}$ in the cultures. Oxidation of acetate with concomitant formation of $\mathrm{H}_{2}$ in the absence of electron acceptors has been observed in acetate-oxidizing methanogenic cocultures $(25,41,53)$. Therefore, if organisms with such a capability were present in our cultures, the $\mathrm{H}_{2}$ threshold concentrations reported here are overestimates of the actual threshold concentrations. Gas leakage through the septum was also of some concern, since the average $\mathrm{H}_{2}$ mixing ratio in the troposphere is $0.56 \mathrm{ppmv}$ (7), and even higher values are generally obtained in microbiological laboratories in which $\mathrm{H}_{2}$ is used for medium preparation and analytical applications. Therefore, the low $\mathrm{H}_{2}$ concentrations $(<0.5$ ppmv) measured for chlororespiring OCRBs cannot be explained by $\mathrm{H}_{2}$ leakage out of the serum bottles. In contrast, one can argue that the actual threshold concentrations might be even lower due to leakage of atmospheric $\mathrm{H}_{2}$ into the culture bottles. $\mathrm{H}_{2}$ threshold concentrations are also temperature dependent $(25,51,53)$. Lee and Zinder (25) demonstrated that there was a 250 -fold increase in the $\mathrm{H}_{2}$ partial pressure in a thermophilic methanogenic coculture over a $60^{\circ} \mathrm{C}$ temperature range. All $\mathrm{H}_{2}$ threshold concentrations in our study were measured by using cultures that were incubated at $25^{\circ} \mathrm{C}$. Hence, the in situ $\mathrm{H}_{2}$ concentrations might be lower than the values reported here.

Another important consideration is that the $\mathrm{H}_{2}$ threshold concentrations are approached asymptotically (Fig. 1). The time required until a steady-state threshold concentration is reached depends on the kinetics of the $\mathrm{H}_{2}$-consuming reactions, and an initial analysis indicated that the $\mathrm{H}_{2}$ consumption kinetics for different chlororespiring organisms can be quite different (unpublished results). The rates of $\mathrm{H}_{2}$ consumption do not influence the final $\mathrm{H}_{2}$ threshold concentrations; however, it is important to verify that the $\mathrm{H}_{2}$ concentrations measured are the end points of $\mathrm{H}_{2}$ consumption.

The results of this study suggest that chlororespiring OCRBs 

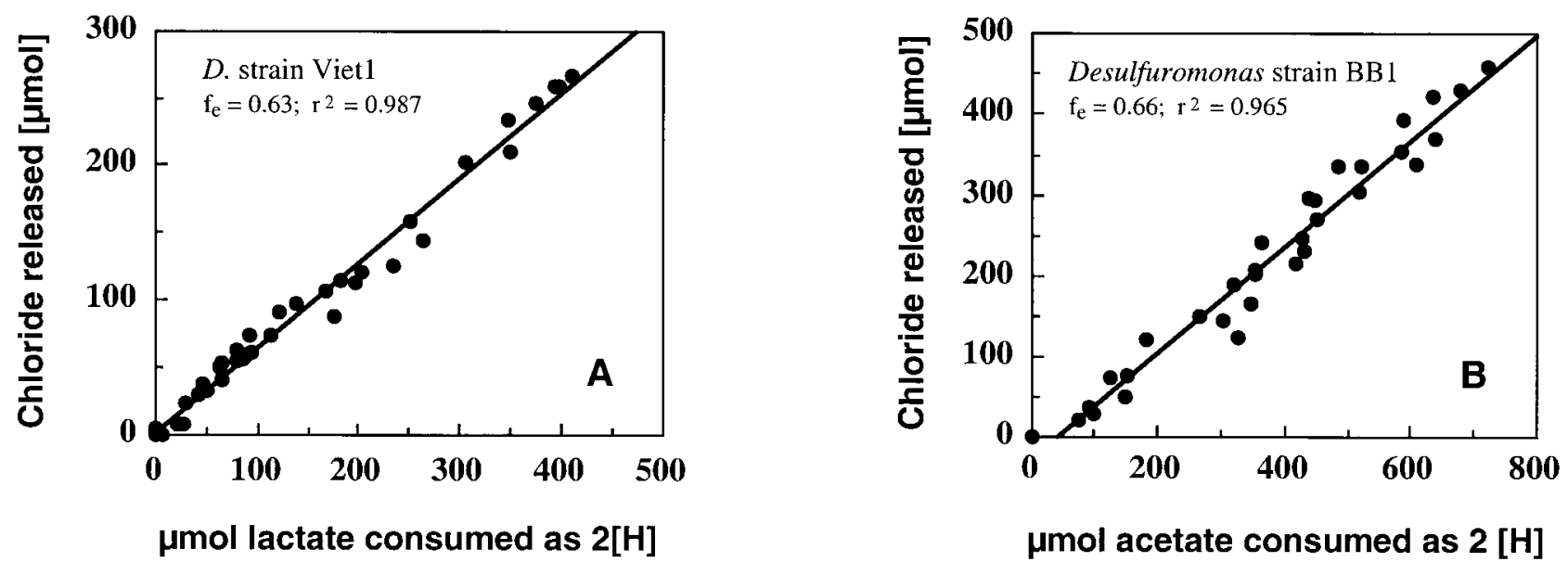

$\mu \mathrm{mol}$ acetate consumed as $2[\mathrm{H}]$
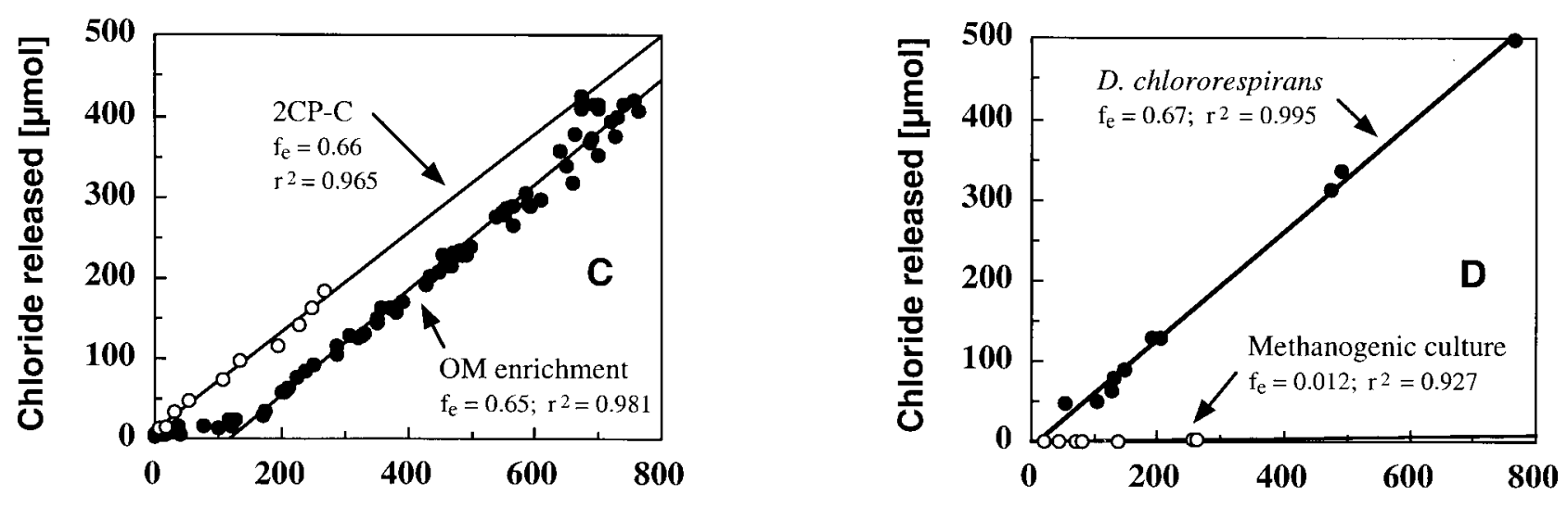

$\mu$ mol acetate or $\mathrm{H}_{2}$ consumed as 2[H]

umol lactate/VFAs consumed as $\mathbf{2}[\mathrm{H}]$

FIG. 2. Graphical determination of $\mathrm{f}_{\mathrm{e}}$ values for dechlorinating cultures, in which the amounts of reducing equivalents $(2[\mathrm{H}])$ generated during oxidation of the electron donor were plotted against the amounts of electron acceptor reduced. The $\mathrm{f}_{\mathrm{e}}$ is indicated by the slope of the regression line. (A) Desulfuromonas sp. strain BB1 (acetate plus PCE). (B) Desulfitobacterium sp. strain Viet1 (lactate plus PCE). (C) OM enrichment culture ( $\mathrm{H}_{2}$ plus 2,5-DCP) and strain 2CP-C (acetate plus 2-CP). (D) Desulfitobacterium chlororespirans (lactate plus 3Cl-4-HBA) and methanogenic enrichment culture (VFA mixture and 3-CP).

are excellent competitors for $\mathrm{H}_{2}$ in anaerobic environments and may be the predominant hydrogenotrophic organisms in environments contaminated with chlorinated compounds. Due to the favorable thermodynamics of reductive dechlorination reactions (Table 1), chlororespirers can maintain concentrations below the concentrations that would allow sulfate reduction or methanogenesis. Hence, chlororespirers can outcompete methanogens and sulfidogens for $\mathrm{H}_{2}$ and should dominate anaerobic environments as long as reductive dechlorination is not limited by the concentration or bioavailability of the chlorinated electron acceptor. As evidence of this we diluted out methanogens and sulfidogens over time from chlororespiring enrichment cultures. Similar observations were made in a continuous stirred-tank reactor fed with benzoate and cis-DCE, in which dechlorination accounted for $42 \%$ of the electrons from the electron donor when the steady-state $\mathrm{H}_{2}$ concentration was only $2 \mathrm{nM}(2.6 \mathrm{ppmv})$ and below the threshold concentration observed for methanogenesis (11 nM) (50).

In field studies, Lovley et al. (34) have demonstrated that $\mathrm{H}_{2}$ threshold concentrations can be used to determine the dominant TEAP in anaerobic subsurface environments. This ap- proach should also be applicable to establishing the presence and activity of chlororespiring OCRBs in situ if nitrate and ferric iron are not present. However, $\mathrm{H}_{2}$ threshold data cannot predict the extent of reductive dechlorination. For example, incomplete halorespiratory dechlorination of PCE to cis-DCE by a hydrogenotrophic dechlorinator results in low $\mathrm{H}_{2}$ threshold concentrations similar to those observed after complete halorespiratory dechlorination to ETH.

Each reductive dechlorination step in the sequential dechlorination of PCE to ETH is associated with a considerable change in free energy, which ranges from -139 to $-173 \mathrm{~kJ}$ mol $^{-1}$ when $\mathrm{H}_{2}$ is the electron donor. Theoretically, each individual step could support chlororespiration, although to date no anaerobic organisms that grow at the expense of $\mathrm{VC}$ have been described and only one organism that uses cis-DCE has been described (35). This is unfortunate, since VC accumulation in contaminated groundwater as a result of reductive dechlorination of PCE is a major concern due to the toxic nature of this compound. The $\mathrm{H}_{2}$ threshold data presented in this paper strongly suggest that VC-respiring organisms are present in river sediments. These findings are encouraging and imply 
TABLE 3. Comparison of measured $\mathrm{f}_{\mathrm{e}}$ values and $\mathrm{f}_{\mathrm{e}}$ values calculated from yield data for different chlororespiring pure cultures

\begin{tabular}{|c|c|c|c|c|c|}
\hline Culture & Electron acceptor & Measured $\mathrm{f}_{\mathrm{s}}^{a}$ & Calculated $\mathrm{f}_{\mathrm{e}}^{b}$ & Measured $f_{e}^{c}$ & Reference \\
\hline Dehalobacter restrictus PER-K23 & PCE & 0.27 & 0.73 & $\mathrm{ND}^{d}$ & 22 \\
\hline Dehalobacter sp. strain TEA & PCE & 0.04 & 0.96 & ND & 48 \\
\hline Dehalospirillum multivorans & PCE & 0.20 & 0.80 & ND & 42 \\
\hline Desulfuromonas ethenica TT4B & PCE & 0.03 & 0.97 & ND & 24 \\
\hline Desulfuromonas sp. strain BB1 & PCE & 0.07 & 0.93 & 0.67 & 30 \\
\hline Dehalococcoides ethenogenes 195 & PCE & 0.46 & 0.54 & ND & 35 \\
\hline Desulfitobacterium sp. strain PCE1 & PCE & 0.22 & 0.78 & ND & 18 \\
\hline Desulfitobacterium chlororespirans Co23 & 3Cl-4-HBA & 0.29 & 0.71 & 0.67 & 39 \\
\hline Desulfomonile tiedjei DCB-1 & 3-CBA & 0.24 & 0.76 & ND & 12 \\
\hline Strain $2 \mathrm{CP}-\mathrm{C}$ & $2-\mathrm{CP}$ & 0.32 & 0.68 & 0.66 & 5 \\
\hline
\end{tabular}

${ }^{a} \mathrm{f}_{\mathrm{s}}$ values were calculated from reported yield data for each culture grown under chlororespiring conditions. The molecular formula for cells used was $\mathrm{C}_{5} \mathrm{H}_{7} \mathrm{O}_{2} \mathrm{~N}$.

${ }^{b} \mathrm{f}_{\mathrm{e}}$ values were calculated from the relationship $\mathrm{f}_{\mathrm{e}}=1-\mathrm{f}_{\mathrm{s}}$.

${ }^{c} \mathrm{f}_{\mathrm{e}}$ values were measured as described in Materials and Methods.

${ }^{d} \mathrm{ND}$, not determined.

that chloroethene-contaminated anaerobic sites could be completely detoxified through stimulation of chlororespiring OCRBs.

Chlororespiring cultures consistently have $\mathrm{f}_{\mathrm{e}}$ values between 0.63 and 0.71 , which are considerably lower than the $f_{e}$ values obtained for sulfidogenic cultures (0.84 to 0.94$)$ (47) or methanogenic cultures (0.95) (10). Chlororespiring cultures, however, have higher $\mathrm{f}_{\mathrm{e}}$ values than aerobic cultures $(0.57)(21)$ or denitrifying cultures (0.54) (4) that utilize the same electron donor, acetate. Energetically, this is sensible since the change in Gibbs free energy $\left(\Delta G^{0^{\prime}}\right)$ for chlororespiration is between the values for sulfate reduction and denitrification (Table 1). The $\Delta G^{0^{\prime}}$ for chlororespiration is in the same range as the energy available from dissimilatory nitrate reduction to ammonia. In fact, the $\mathrm{f}_{\mathrm{e}}$ values that have been measured for ammonifiers are around $0.6(4)$ and are slightly lower than the $f_{e}$ values determined in this study for chlororespiring OCRBs. This verifies that there is a thermodynamic basis to $\mathrm{f}_{\mathrm{e}}$, with more energetically favorable electron-accepting reactions having lower $\mathrm{f}_{\mathrm{e}}$ values, as described by McCarty (36). The expectation is that the $f_{e}$ increases according to the following sequence of electron acceptors: $\mathrm{O}_{2}<\mathrm{NO}_{3}{ }^{-}<$chlorinated compounds $<\mathrm{SO}_{4}{ }^{-2}<\mathrm{CO}_{2}$.

The $f_{e}$ values determined for chlororespiring cultures indicate that the reductive dechlorination reactions are indeed respiratory TEAPs, since other possible mechanisms coupling this process to growth would not exhibit similar $f_{e}$ values. For example, it is possible that some microorganisms fortuitously catalyze reductive dechlorination reactions (cometabolism) and channel a small fraction of the available electrons from the electron donor toward reductive dechlorination without gaining energy from this process. Cometabolic dechlorination, however, is usually characterized by a low $f_{e}$ value in methanogenic and acetogenic systems. This low $f_{e}$ value associated with fortuitous cometabolic reactions occurs because no electron transport or proton translocation forming a proton motive force to drive membrane-associated ATP formation occurs. Since only respiratory processes have such mechanisms for ATP generation, less energy would be available for growth in an organism lacking this ability. Therefore, the energy that could be derived from reductive dechlorination would be lost (as heat), and the microbial growth yields $\left(f_{s}\right)$ would be smaller. Table 3 shows $f_{s}$ values calculated from yield data obtained for various pure cultures by utilizing chlorinated compounds as sole terminal electron acceptors. The $\mathrm{f}_{\mathrm{e}}$ was calculated $\left(1-f_{s}\right)$ for each culture, and it is evident that most of the values are in agreement with the measured $f_{e}$ values for chlo- rorespiratory OCRBs reported in this study. For example, there is good agreement between the measured $f_{e}$ values for strains 2CP-C (0.66) and $\mathrm{Co} 23$ (0.67) and the $\mathrm{f}_{\mathrm{e}}$ values calculated from yield data ( 0.68 and 0.71 , respectively). The exceptions to this agreement are the $\mathrm{f}_{\mathrm{e}}$ values calculated from the yield data obtained for Desulfuromonas sp. strain BB1, Desulfuromonas ethenica TT4B, and Dehalobacter sp. strain TEA (Table 3), which are considerably higher than would be predicted. It has been shown, however, that Desulfuromonas sp. strain BB1, a close relative of Desulfuromonas ethenica TT4B, has a measured $f_{e}$ of 0.66 (Fig. 2). There is also some inconsistency between the closely related organisms Dehalobacter sp. strain TEA $\left(\mathrm{f}_{\mathrm{e}}=0.96\right)$ and Dehalobacter restrictus PER-K23 $\left(f_{e}=0.73\right)$. This disagreement in the data has some reasonable explanations. Measurement of $\mathrm{H}_{2}$ can be difficult, or other potential electron donors (e.g., yeast extract) were not included in the calculation; therefore, a complete accounting of the mass balance was potentially affected. Another concern is that some of the yield data were determined after growth had stopped and the stationary phase was reached. Preliminary studies with strain BB1 have shown that electron donor oxidation and reductive dechlorination continue in the stationary phase (30). This creates an uncoupling of growth from chlororespiration and leads to underestimation of the maximum growth yield (and therefore of $f_{s}$ ) and overestimation of $f_{e}$, depending on when the growth yields were determined. Hence, $\mathrm{f}_{\mathrm{s}(\max )}$ should be determined during the exponential growth phase. Generally, chlororespiring OCRBs are grown with the chlorinated electron acceptor and the electron donor present in excess. Under substrate-sufficient conditions consumption of an energy source and complete absence of growth have been observed, indicating that the energy generated in catabolic reactions (reductive dechlorination of PCE) was uncoupled from anabolism (26). As a result, $\mathrm{f}_{\mathrm{e}}$ values calculated from yield data $\left(f_{s}\right)$ are overestimates. Hence, it is recommended that $\mathrm{f}_{\mathrm{e}}$ be measured as described in this study.

In conclusion, our findings suggest that $\mathrm{f}_{\mathrm{e}}$ values and $\mathrm{H}_{2}$ threshold concentrations may be used to help predict whether chlororespiration is the predominant TEAP in pure and mixed cultures of OCRBs. When combined, the two values can distinguish anaerobic cometabolic dechlorination processes from chlororespiration. Since electron transport mechanisms of OCRBs have not been well-elucidated, further research should focus on identifying components of the respiratory chain in order to conclusively link $\mathrm{f}_{\mathrm{e}}$ values and $\mathrm{H}_{2}$ threshold concentrations to halorespiratory processes. 


\section{ACKNOWLEDGMENTS}

This research was supported in part by a Feodor-Lynen fellowship from the Alexander von Humboldt-Stiftung to F.E.L. and by Department of Energy grant DE-FG07-96ER62319 to F.E.L. and J.M.T., and additional support was provided by the SERDP Bioconsortium, by a University of Illinois Research Board grant to R.A.S., and by National Science Foundation grant DEB9120006 through the Center for Microbial Ecology.

We thank John A. Breznak, Jared R. Leadbetter, and Sayed Hashsham for help with the initial hydrogen threshold measurements. Kirsti M. Ritalahti is gratefully acknowledged for critically reading the manuscript.

\section{REFERENCES}

1. Bergmann, J. G., and J. Sanik, Jr. 1957. Determination of trace amounts of chlorine in naphtha. Anal. Chem. 29:241-243.

2. Breznak, J. A. 1994. Acetogenesis from carbon dioxide in termite guts, p. 303-330. In H. L. Drake (ed.), Acetogenesis. Chapman \& Hall, Inc., New York, N.Y.

3. Breznak, J. A., and R. N. Costilow. 1993. Physicochemical factors of growth, p. 137-154. In P. Gerhardt, R. G. E. Murray, W. A. Wood, and N. R. Krieg (ed.), Methods for general and molecular bacteriology. American Society for Microbiology, Washington, D.C.

4. Carley, B. N., and D. S. Mavinic. 1991. The effects of external carbon loading on nitrification and denitrification of a high-ammonia landfill leachate. Res. J. Water Pollut. Control Fed. 63:51.

5. Cole, J. R., A. L. Cascarelli, W. W. Mohn, and J. M. Tiedje. 1994. Isolation and characterization of a novel bacterium growing via reductive dehalogenation of 2-chlorophenol. Appl. Environ. Microbiol. 60:3536-3542.

6. Cole, J. R., B. Z. Fathepure, and J. M. Tiedje. 1995. Tetrachloroethene and 3-chlorobenzoate activities are co-induced in Desulfomonile tiedjei DCB-1. Biodegradation 6:167-172.

7. Conrad, R., M. Aragno, and W. Seiler. 1983. The inability of hydrogen bacteria to utilize atmospheric hydrogen is due to threshold and affinity for hydrogen. FEMS Microbiol. Lett. 18:207-210.

8. Conrad, R. 1996. Soil microorganisms as controllers of atmospheric trace gases $\left(\mathrm{H}_{2}, \mathrm{CO}, \mathrm{CH}_{4}, \mathrm{OCS}, \mathrm{N}_{2} \mathrm{O}\right.$, and NO). Microbiol. Rev. 60:609-640.

9. Cord-Ruwisch, R., H.-J. Seitz, and R. Conrad. 1988. The capacity of hydrogenotrophic anaerobic bacteria to compete for traces of hydrogen depends on the redox potential of the terminal electron acceptor. Arch. Microbiol. 149: 350-357.

10. Criddle, C. S., L. M. Alvarez, and P. L. McCarty. 1991. Microbial processes in porous media, p. 641-691. In J. Bear and M. Y. Corapcioglu (ed.), Transport processes in porous media. Kluwer Academic Publishers, Dordrecht, The Netherlands.

11. Deweerd, K. A., L. Mandelco, R. S. Tanner, C. R. Woese, and J. M. Suflita. 1991. Desulfumonile tiedjei gen. nov. and sp. nov., a novel anaerobic, dehalogenating, sulfate-reducing bacterium. Arch. Microbiol. 154:23-30.

12. Deweerd, K. A., F. Concannon, and J. M. Suflita. 1991. Relationship between hydrogen consumption, dehalogenation, and the reduction of sulfur oxyanions by Desulfomonile tiedjei. Appl. Environ. Microbiol. 57:1929-1934.

13. Dolfing, J., and B. K. Harrison. 1992. Gibbs free energy of formation of halogenated aromatic compounds and their potential role as electron acceptors in anaerobic environments. Environ. Sci. Technol. 26:2213-2218.

14. Dolfing, J., and D. B. Janssen. 1994. Estimates of Gibbs free energies of formation of chlorinated aliphatic compounds. Biodegradation 5:21-28.

15. Dolfing, J., and J. E. M. Beurskens. 1995. The microbial logic and environmental significance of reductive dehalogenation, p. 143-206. In J. Gwynfryn Jones (ed.), Advances in microbial ecology, vol. 14. Plenum Press, New York, N.Y.

16. Fathepure, B. Z., and S. A. Boyd. 1988. Reductive dechlorination of perchloroethylene and the role of methanogens. FEMS Microbiol. Lett. 49:149156.

17. Fetzner, S. 1998. Bacterial dehalogenation. Appl. Microbiol. Biotechnol. 50:633-657.

18. Gerritse, J., V. Renard, T. M. Pedro Gomes, P. A. Lawson, M. D. Collins, and J. C. Gottschal. 1996. Desulfitobacterium sp. strain PCE1, an anaerobic bacterium that can grow by reductive dechlorination of tetrachloroethene or ortho-chlorinated phenols. Arch. Microbiol. 165:132-140.

19. Gossett, J. M. 1987. Measurement of Henry's Law constants for C1 and C2 chlorinated hydrocarbons. Environ. Sci. Technol. 21:202-208.

20. Heijnen, J. J., and J. P. van Dijken. 1992. In search of a thermodynamic description of biomass yields for the chemotrophic growth of microorganisms. Biotechnol. Bioeng. 39:833-858.

21. Heijnen, J. J., M. C. M. van Loosdrecht, and L. Tijhuis. 1992. A black box mathematical model to calculate auto- and heterotrophic biomass yields based on Gibbs energy dissipation. Biotechnol. Bioeng. 40:1139-1154.

22. Holliger, C., D. Hahn, H. Harmsen, W. Ludwig, W. Schumacher, B. Tindall, F. Vazquez, N. Weiss, and A. J. B. Zehnder. 1998. Dehalobacter restrictus gen. nov. and sp. nov., a strictly anaerobic bacterium that reductively dechlorinates tetra- and trichloroethene in an anaerobic respiration. Arch. Microbiol. 169:313-321.

23. Jablonski, P. E., and J. G. Ferry. 1992. Reductive dechlorination of trichloroethylene by the $\mathrm{CO}$-reduced $\mathrm{CO}$ dehydrogenase enzyme complex from Methanosarcina thermophila. FEMS Microbiol. Lett. 96:55-60.

24. Krumbolz, L. R., R. Sharp, and S. Fishbain. 1996. A freshwater anaerobe coupling acetate oxidation to tetrachloroethene dehalogenation. Appl. Environ. Microbiol. 62:4108-4113.

25. Lee, M. J., and S. H. Zinder. 1988. Hydrogen partial pressures in a thermophilic acetate-oxidizing methanogenic coculture. Appl. Environ. Microbiol. 54:1457-1461.

26. Liu, Y. 1998. Energy uncoupling in microbial growth under substrate-sufficient conditions. Appl. Microbiol. Biotechnol. 49:500-505.

27. Löffler, F. E., R. A. Sanford, and J. M. Tiedje. 1996. Initial characterization of a reductive dehalogenase from Desulfitobacterium chlororespirans Co23. Appl. Environ. Microbiol. 62:3809-3813.

28. Löffler, F. E., J. E. Champine, K. M. Ritalahti, S. J. Sprague, and J. M. Tiedje. 1997. Complete reductive dechlorination of 1,2-dichloropropane by anaerobic bacteria. Appl. Environ. Microbiol. 63:2870-2875.

29. Löffler, F. E., K. M. Ritalahti, and J. M. Tiedje. 1997. Dechlorination of chloroethenes is inhibited by 2-bromoethanesulfonate in the absence of methanogens. Appl. Environ. Microbiol. 63:4982-4985.

30. Löffler, F. E., J. Li, J. W. Urbance, and J. M. Tiedje. 1998. Characterization of strain BB1, a tetrachloroethene (PCE)-dechlorinating anaerobe, abstr. Q-177, p. 450. In Abstracts of the 98th General Meeting of the American Society for Microbiology 1998. American Society for Microbiology, Washington, D.C.

31. Löffler, F. E., B. Z. Fathepure, P. Adriaens, and J. M. Tiedje. 1999. Evidence for the presence of chlororespiring bacteria in a chloroethene contaminated aquifer. In Abstracts of the Fifth International Symposium on In Situ and On-Site Bioremediation. Batelle, San Diego, Calif.

32. Lovley, D. R. 1985 . Minimum threshold for hydrogen metabolism in methanogenic bacteria. Appl. Environ. Microbiol. 49:1530-1531.

33. Lovley, D. R., and S. Goodwin. 1988. Hydrogen concentrations as an indicator of the predominant terminal electron-accepting reactions in aquatic sediments. Geochim. Cosmochim. Acta 52:2993-3003.

34. Lovley, D. R., F. H. Chapelle, and J. C. Woodward. 1994. Use of dissolved $\mathrm{H}_{2}$ concentrations to determine distribution of microbially catalyzed redox reactions in anaerobic groundwater. Environ. Sci. Technol. 28:12051210.

35. Maymó-Gatell, X., Y.-T. Chien, J. M. Gossett, and S. H. Zinder. 1997. Isolation of a bacterium that reductively dechlorinates tetrachloroethene to ethene. Science 276:1568-1571.

36. McCarty, P. L. 1971. Energetics and bacterial growth, p. 495-531. In J. Faust and J. V. Hunter (ed.), Organic compounds in aquatic environments. Marcel Dekker, Inc., New York, N.Y.

37. Mohn, W. W., and J. M. Tiedje. 1990. Strain DCB-1 conserves energy for growth from reductive dechlorination coupled to formate oxidation. Arch. Microbiol. 153:267-271.

38. Sanford, R. A. 1996. Characterization of microbial populations in anaerobic food webs that reductively dechlorinate chlorophenols. Ph.D. thesis. Michigan State University, East Lansing.

39. Sanford, R. A., J. R. Cole, F. E. Löffler, and J. M. Tiedje. 1996. Characterization of Desulfitobacterium chlororespirans sp. nov., which grows by coupling the oxidation of lactate to the reductive dechlorination of 3-chloro-4hydroxybenzoate. Appl. Environ. Microbiol. 62:3800-3808.

40. Sanford, R. A., and J. M. Tiedje. 1996. Chlorophenol dechlorination and subsequent degradation in denitrifying microcosms fed low concentrations of nitrate. Biodegradation 7:425-434.

41. Schnürer, A., B. Schink, and B. H. Svesson. 1996. Clostridium ultunense sp. nov., a mesophilic bacterium oxidizing acetate in syntrophic association with a hydrogenotrophic methanogenic bacterium. Int. J. Syst. Bacteriol. 46:11451152.

42. Scholz-Muramatsu, H., A. Neumann, M. Meßmer, E. Moore, and G. Diekert. 1995. Isolation and characterization of Dehalospirillum multivorans gen. nov., sp. nov., a tetrachloroethene-utilizing, strictly anaerobic bacterium. Arch. Microbiol. 163:48-56.

43. Shelton, D. R., and J. M. Tiedje. 1984. Isolation and partial characterization of bacteria in an anaerobic consortium that mineralizes 3-chlorobenzoic acid. Appl. Environ. Microbiol. 48:840-848.

44. Terzenbach, D. P., and M. Blaut. 1994. Transformation of tetrachloroethylene to trichloroethylene by homoacetogenic bacteria. FEMS Microbiol. Lett. 123:213-218.

45. Thauer, R. K., K. Jungermann, and K. Decker. 1977. Energy conservation in chemotrophic anaerobes. Bacteriol. Rev. 41:100-180.

46. Townsend, G. T., and J. M. Suflita. 1996. Characterization of chloroethylene dehalogenation by cell extracts of Desulfomonile tiedjei and its relationship to chlorobenzoate dehalogenation. Appl. Environ. Microbiol. 62:2850-2853.

47. Widdel, F. 1988. Microbiology and ecology of sulfate- and sulfur-reducing bacteria, p. 469-586. In A. J. B. Zehnder (ed.), Biology of anaerobic microorganisms. John Wiley and Sons, Inc., London, United Kingdom. 
48. Wild, A., R. Hermann, and T. Leisinger. 1996. Isolation of an anaerobic bacterium which reductively dechlorinates tetrachloroethene and trichloroethene. Biodegradation 7:507-511.

49. Wilhelm, E., R. Battino, and R. J. Wilcock. 1977. Low pressure solubility of gases in liquid water. Chem. Rev. 77:219-262.

50. Yang, Y., and P. L. McCarty. 1998. Competition for hydrogen within a chlorinated solvent dehalogenating anaerobic mixed culture. Environ. Sci. Technol. 32:3591-3597.
51. Zehnder, A. J. B., and W. Stumm. 1988. Geochemistry and biogeochemistry of anaerobic habitats, p. 1-38. In A. J. B. Zehnder (ed.), Biology of anaerobic microorganisms. John Wiley \& Sons, Inc., New York, N.Y.

52. Zinder, S. H. 1993. Physiological ecology of methanogens, p. 128-206. In J. G. Ferry (ed.), Methanogenesis. Chapman \& Hall, Inc., New York, N.Y.

53. Zinder, S. H. 1994. Syntrophic acetate oxidation and "reversible acetogenesis," p. 386-415. In H. L. Drake (ed.), Acetogenesis. Chapman \& Hall, Inc., New York, N.Y. 